\title{
Intra- versus retroplacental hematomas: a retrospective case-control study on pregnancy outcomes
}

\author{
Johannes Ott ${ }^{1}$, Philipp Pecnik ${ }^{2,3}$, Regina Promberger ${ }^{2,4^{*}}$, Sophie Pils ${ }^{1}$, Julia Binder ${ }^{2}$ and Kinga M. Chalubinski ${ }^{2}$
}

\begin{abstract}
Background: Intrauterine hematomas are a common pregnancy complication. The literature lacks studies about outcomes based on hematoma localization. Thus, we aimed to compare pregnancies complicated by an intraplacental hematoma to cases with a retroplacental hematoma and to a control group.

Methods: In a retrospective case-control study, 32 women with an intraplacental hematoma, 199 women with a retroplacental hematoma, and a control group consisting of 113 age-matched women with no signs of placental abnormalities were included. Main outcome measures were pregnancy complications.
\end{abstract}

Results: Second-trimester miscarriage was most common in the intraplacental hematoma group (9.4\%), followed by women with a retroplacental hematoma (4.2\%), and controls ( $0 \% ; p=0.007)$. The intraplacental hematoma group revealed the highest rates for placental insufficiency, intrauterine growth retardation, premature preterm rupture of membranes, preterm labor, preterm delivery $<37$ weeks, and early preterm delivery $<34$ weeks $(p<0.05)$, followed by the retroplacental hematoma group. When tested in multivariate models, intraplacental hematomas were independent predictors for placental insufficiency $(\beta=4.19, p<0.001)$ and intrauterine growth restriction $(B=1.44, p=0.035)$. Intrauterine fetal deaths occurred only in women with a retroplacental hematoma $(p=0.042)$.

Conclusions: Intra- and retroplacental hematomas have different risk profiles for the affected pregnancy and act as independent risk factors.

Keywords: Intraplacental hematoma, Retroplacental hematoma, Intrauterine fetal death, Placental insufficiency, Pregnancy complications

\section{Background}

Intrauterine hematomas are a common pregnancy complication, and can occur at any time during the entire pregnancy, with an associated appearance of obstetrical bleeding in $5-25 \%$ in the first trimester, putting mother and child at risk [1]. The incidence of hematomas in the first trimester is reportedly $4-22 \%$, with smaller hematomas often occurring in the first trimester, whereas larger intrauterine masses are more common in the second trimester [2]. Studies and reviews have shown that both early and late hematomas are associated with a higher

\footnotetext{
* Correspondence: regina.promberger@chello.at

${ }^{2}$ Department of Obstetrics and Fetomaternal Medicine, Medical University of Vienna, Waehringer Guertel 18-20, 1090 Vienna, Vienna, Austria

${ }^{4}$ Department of Obstetrics and Gynecology, Saint John of God Hospital

Eisenstadt, Johannes-von-Gott Platz 1, 7000 Eisenstadt, Burgenland, Austria

Full list of author information is available at the end of the article
}

rate of adverse events, such as vaginal hemorrhage, miscarriage, early delivery, pregnancy-induced hypertension, pre-eclampsia, gestational diabetes, intrauterine growth restriction, and even stillbirth [2-5]. The term "intrauterine hematoma" encompasses several entities that commonly include retroplacental, subchorionic, and subamniotic hematomas. As retroplacental and marginal hematomas are most frequent, most existing studies about the outcome of pregnancies with hematomas refer to these two entities, with intraplacental hematomas sparsely evaluated.

Placental bleedings can be classified according to their as retroplacental, subchorionic, subamniotic, or intraplacental hematomas. The latter are rare and the literature on this entity is scarce. Notably, in a recent histopathological study, rounded intraplacental hematomas revealed 
morphological features than other than parabasally located intervillous thrombohematomas [6]. Sonographically, intraplacental hematomas are located in the intervillous cavity of the placenta, whereas retroplacental hematomes are located between the basal plate and myometrium, lifting the placental parenchyma toward the amniotic cavity [7]. This suggests that intraplacental hematomas are a separate entity, and validates our clinical experience that intraplacental hematomas are associated with an extraordinarily increased risk of fetal and maternal adverse events. Thus, we believe that this risk exceeds that of the other types of intrauterine hematomas. To date, the literature focused on intrauterine hematomas is general in nature, and no studies have evaluated the outcomes of intraplacental hematomas separately. Thus, we aimed to evaluate neonatal and obstetric outcomes in all patients with an intraplacental hematoma who had been diagnosed between 2006 and 2012 at the Department of Obstetrics and Fetomaternal Medicine of the Medical University of Vienna, Austria. These 32 cases were compared to (i) women who suffered from a retroplacental hematoma, and (ii) an aged-matched control group that included women with no signs of placental abnormalities.

\section{Methods}

\section{Patient population and study design}

In a retrospective study, all women with an intraplacental hematoma $(n=32)$ and a retroplacental hematoma $(n=199)$ who had delivered at the Department of Obstetrics and Fetomaternal Medicine of the Medical University of Vienna, Austria, between August 2006 and January 2012, were identified. As a control group, 113 age-matched women with no signs of placental abnormalities who had also delivered at our department within the same time period were also included. For selection of controls, a large database including all deliveries at the department was used and the procedure was performed by "case-control-matching" in SPSS 17.0 software (SPSS Inc., 1989-2009). Retrospective chart review was performed using the PIA Fetal Database software (GE-Viewpoint, Wessling, Germany).

All hematomas were diagnosed during pregnancy via ultrasound in the course of either a routine examination (first or second trimester screening) or an examination for vaginal bleeding. All hematoma-specific ultrasound examinations were performed by one highly experienced operator (K.M.C.), using commercially available, real-time equipment. In detail, a Toshiba Power Vision (Toshiba, Tokyo, Japan) ultrasound machine was used until 2007, and a Toshiba Aplio MX machine from 2007 to 2012. For each patient, the whole placenta was scanned in a systematic fashion, using both gray-scale ultrasound and color-flow mapping. Standard $3.75 \mathrm{MHz}$ linear or sector transducers were used for abdominal ultrasound and a $7.5 \mathrm{MHz}$ transducer was used transvaginally. Doppler power settings were at the level approved for fetal use. The diagnosis of an intrauterine hematoma was established during either routine first- and second-trimester screening or during a non-routine ultrasound for vaginal bleeding. Intraplacental hematomas were defined as sonographically diagnosed hypoechoic masses, with no signs of blood flow, located in the intervillous cavity of the placenta (Fig. 1a and b). In the present analysis, this entity also included massive subchorionic hematomas [7]. Retroplacental hematomas were defined as a hypoechoic area between the basal plate and myometrium, lifting the placental parenchyma toward the amniotic cavity (Fig. 1c and d) [7].

In patients of the control group, intrauterine hematomas had been excluded during first- and secondtrimester screenings, as well as in the course of the final ultrasound before delivery, i.e., after the onset of spontaneous labor or 1 day before elective Cesarean section.

Patients with an intrauterine hematoma underwent regular follow-up examinations, including gray-scale and Doppler sonography on an individual basis, at least every 2 weeks. The study was approved by the Institutional Review Board of the Medical University of Vienna (IRB number: 1681/2014). Neither written nor verbal informed consent is unnecessary in retrospective studies according to the Ethics Committee of the Medical University of Vienna and was, thus, not obtained.

\section{Parameters analyzed}

As outcome parameters, we focused on the incidences of the following pregnancy complications: pregnancyinduced hypertension, defined as a blood pressure of $140 \mathrm{mmHg}$ systolic or higher or $90 \mathrm{mmHg}$ diastolic or higher that occurred after 20 weeks of gestation in a woman with previously normal blood pressure with no proteinuria or other signs of organ dysfunction [8]; preeclampsia, defined as blood pressure of $140 \mathrm{mmHg}$ systolic or higher or $90 \mathrm{mmHg}$ diastolic or higher that occurred after 20 weeks of gestation in a woman with previously normal blood pressure, and with proteinuria, defined as urinary excretion of $0.3 \mathrm{~g}$ protein or higher in a 24-h urine specimen [9]; placental insufficiency, characterized by the presence of fetal growth restriction, reduced amniotic fluid, and impaired fetal oxygenation and which demonstrated pathological Doppler indices in the umbilical (UA) and uterine arteries [10]. Reference ranges for Doppler parameters were based on the observations of Schaffer et al. [11]; intrauterine growth retardation, defined as an abdominal circumference $<5$ th percentile, measured at the first visit during the second or early third trimester [12]; intrauterine fetal death; preterm premature rupture of membranes, defined as spontaneous rupture of the membranes prior to the onset of labor 

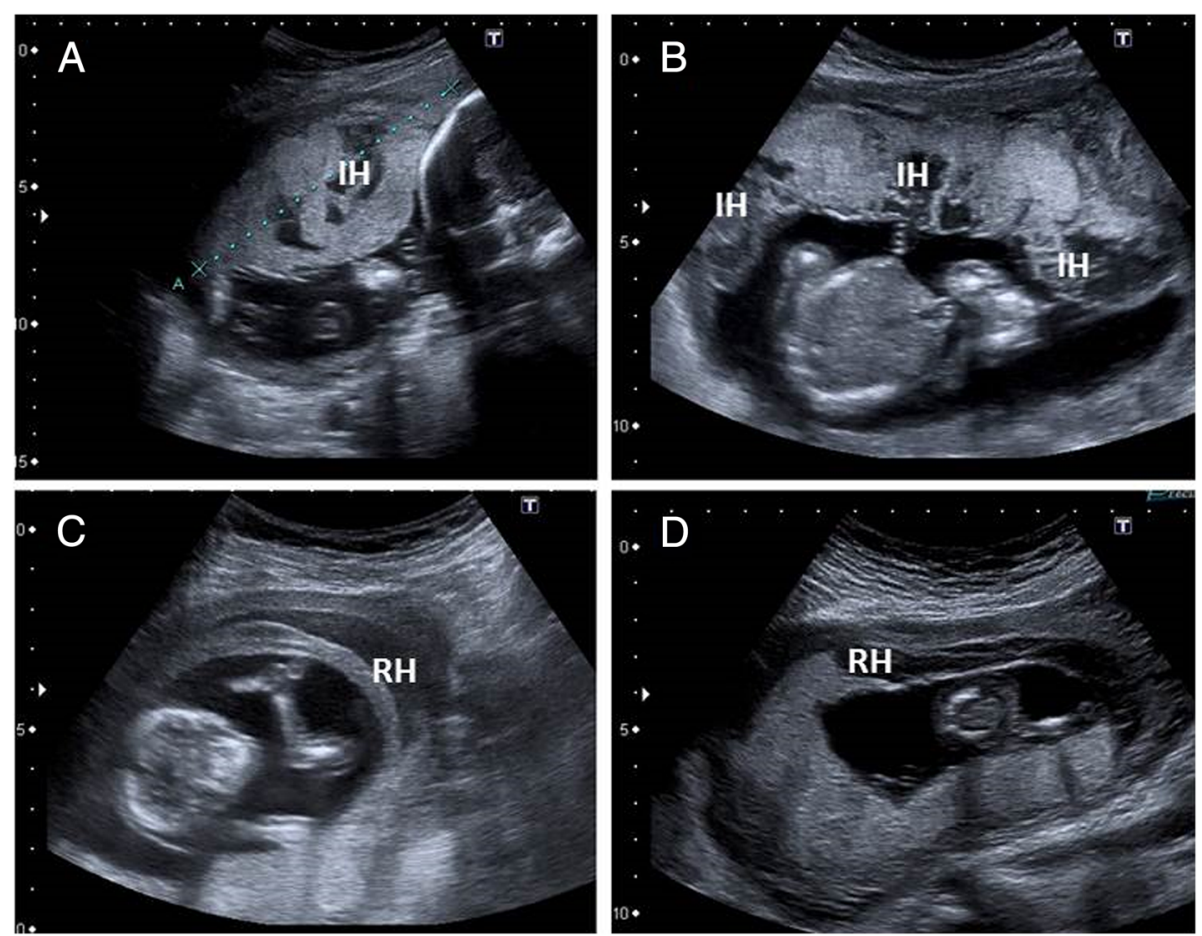

Fig. 1 Sonographic appearance of intraplacental hematomas ("IH", a and $\mathbf{b}$ ) and retroplacental hematomas ("RH", c and $\mathbf{d}$ )

before 37 gestational weeks [13]; preterm labor, defined as regular uterine contractions leading to cervical dilation, effacement, or both, or initial presentation with regular contractions and cervical dilation of at least $2 \mathrm{~cm}$ between $20+0$ weeks of gestation and $36+6$ weeks of gestation preterm delivery $<37$ weeks [14]; early preterm delivery $<34+0$ weeks; and complete placental abruption which was defined as a complete separation of the placental lining from the uterus (in contrast to a retroplacental hematoma). In addition, we included mother's age, parity, the maximum diameter of the hematoma (divided into hematomas $<5 \mathrm{~cm}$ and $\geq 5 \mathrm{~cm}$ ) and whether the woman had suffered from previous intrauterine fetal death (IUFD) as parameters in the predictive models [5].

\section{Statistical analyses}

Nominal variables are reported as numbers and frequencies, and continuous variables with median and interquartile ranges. Differences between groups were tested using ANOVA or Welsch tests, where appropriate, for numeric variables and Chi square or Fisher's exact test for categorical variables. To assess the predictive factors for placental insufficiency and intrauterine growth retardation, multivariate logistic regression models were used. Coefficient estimates, $\beta$ and standard error se( $\beta$ ), as well as corresponding $p$-values, are given for these analyses. Statistical analysis was performed using the
SPSS 17.0 software (SPSS Inc., 1989-2009). Differences were considered statistically significant if $p<0.05$.

\section{Results}

There were no missing data. Basic patient characteristics are shown in Table 1. Notably, the three groups differed in terms of the following parameters $(p<0.05)$. The previous incidence of intrauterine fetal death was highest among women with an intraplacental hematoma (12.5\%), followed by those with a retroplacental hematoma (2.5\%). Chorionic villous sampling preceded an intrauterine hematoma in $6.3 \%(n=2)$ compared to none in the retroplacental hematoma group. Retroplacental hematomas were diagnosed significantly earlier (median 13 weeks; interquartile range, 12-21) than intraplacental hematomas (median 24 weeks; interquartile range, 22-29; $p<0.001$ ).

Maximum hematoma diameter was as follows: a hematoma exceeding $5 \mathrm{~cm}$ of maximum diameter was found in $14 / 29$ intraplacental cases $(48.3 \%)$ compared to $33 / 114(28.9 \%)$ in retroplacental cases $(p=0.041)$.

In the intraplacental hematoma, retroplacental hematoma, and control groups, ten (31.3\%), 71 (59.7\%), and 4 (3.5\%) women, respectively, suffered from vaginal bleeding at least once during the course of their pregnancy $(p<0.001)$. The incidence of second-trimester miscarriage was highest in the intraplacental hematoma group $(n=3,9.4 \%)$, followed by women with a 
Table 1 Patient characteristics

\begin{tabular}{|c|c|c|c|c|}
\hline & $\begin{array}{l}\text { Intraplacental hematoma } \\
(n=32)\end{array}$ & $\begin{array}{l}\text { Retroplacental hematoma } \\
(n=119)\end{array}$ & $\begin{array}{l}\text { Controls } \\
(n=113)\end{array}$ & $p$ \\
\hline Age (years) ${ }^{a}$ & $31(27 ; 35)$ & $31(26 ; 34)$ & $31(27 ; 36)$ & 0.394 \\
\hline Parity $(n)^{a}$ & $1(0 ; 2)$ & $2(0 ; 2)$ & $2(0 ; 2)$ & 0.486 \\
\hline Previous PIH & 0 & $3(2.5)$ & 0 & 0.348 \\
\hline Previous IUGR ${ }^{\mathrm{b}}$ & $1(3.1)$ & $1(0.8)$ & $6(5.3)$ & 0.138 \\
\hline Previous preeclampsiab & $1(3.1)$ & $3(2.5)$ & $1(0.9)$ & 0.534 \\
\hline Previous IGDM ${ }^{b}$ & $1(3.1)$ & $4(3.4)$ & $2(1.8)$ & 0.653 \\
\hline Previous preterm delivery ${ }^{b}$ & $3(9.4)$ & $4(3.4)$ & $7(6.2)$ & 0.287 \\
\hline Previous IUFD ${ }^{b}$ & $4(12.5)$ & $3(2.5)$ & 0 & 0.001 \\
\hline Chorionic villous sampling ${ }^{b}$ & $2(6.3)$ & 0 & $1(0.9)$ & 0.039 \\
\hline $\mathrm{IGDM}^{\mathrm{b}}$ & $3(9.4)$ & $22(18.5)$ & $26(23.0)$ & 0.219 \\
\hline Gestational age at diagnosis (completed weeks) ${ }^{a}$ & $24(22-29)$ & $13(12-21)$ & - & $<0.001$ \\
\hline
\end{tabular}

Data are provided as ${ }^{a}$ median (interquartile range) or ${ }^{b} \mathrm{n}(\%)$

Abbreviations: PIH pregnancy induced hypertension, IUGR intrauterine growth restriction, IGDM insulin-dependent gestational diabetes mellitus, IUFD intrauterine fetal death

retroplacental hematoma $(n=5,4.2 \%)$ and controls $(n=0 ; p=0.007)$.

The latter patients were excluded from the subsequent analyses on pregnancy outcomes, since data on the evaluated outcome parameters were available only from week $24+0$ onwards. In short, the intraplacental hematoma group $(p<0.05)$ revealed significantly higher rates of placental insufficiency, intrauterine growth retardation, premature preterm rupture of membranes, preterm labor, preterm delivery $<37$ weeks, and early preterm delivery $<$ 34 weeks (Table 2). These findings were associated with differences in gestational age at delivery, birth weight, and rates of Cesarean section between the groups $(p<0.001)$. IUFD occurred only in women with retroplacental hematoma. None of the affected fetuses revealed any birth defects. Median gestational age at diagnosis of IUFD was 24 completed weeks (IQR 23-25). For all of these outcome parameters, women with retroplacental hematoma were still at increased risk compared to the controls. However, intrauterine fetal deaths occurred only in the retroplacental hematoma group $(p=0.042)$.

In a next step, we tested several parameters for the prediction of placental insufficiency and intrauterine growth restriction (Table 3). For both outcome parameters, retroplacental hematomas significantly increased the risk for the development of the complication. The presence of a retroplacental hematoma outweighed all other tested parameters apart from previous IUGR as a predictor of its recurrence. When performing a similar analysis for early preterm delivery $<34+0$ weeks

Table 2 Pregnancy outcomes. Patients with second-trimester miscarriage were excluded from these analyses

\begin{tabular}{|c|c|c|c|c|}
\hline & $\begin{array}{l}\text { Intraplacental hematoma } \\
(n=29)\end{array}$ & $\begin{array}{l}\text { Retroplacental hematoma } \\
(n=114)\end{array}$ & $\begin{array}{l}\text { Controls } \\
(n=113)\end{array}$ & $p$ \\
\hline Pregnancy-induced hypertension ${ }^{\mathrm{b}}$ & $4(13.8)$ & $8(7.0)$ & $12(10.6)$ & 0.377 \\
\hline Preeclampsia $^{\mathrm{b}}$ & $2(6.9)$ & $3(2.6)$ & $9(8.0)$ & 0.171 \\
\hline Placental insufficiency ${ }^{\mathrm{b}}$ & $9(31.0)$ & $11(9.6)$ & $1(0.9)$ & $<0.001$ \\
\hline Intrauterine growth retardation ${ }^{\mathrm{b}}$ & $8(27.6)$ & $14(12.3)$ & $10(8.8)$ & 0.034 \\
\hline Intrauterine fetal death ${ }^{b}$ & 0 & $6(5.3)$ & 0 & 0.042 \\
\hline Preterm premature rupture of membranes ${ }^{b}$ & $2(6.9)$ & $29(25.4)$ & $14(12.4)$ & 0.012 \\
\hline Preterm labor ${ }^{b}$ & $8(27.6)$ & $26(22.8)$ & $6(5.3)$ & $<0.001$ \\
\hline Preterm delivery ${ }^{\mathrm{b}}$ & $15(51.7)$ & $34(29.8)$ & $11(9.7)$ & $<0.001$ \\
\hline Early preterm delivery $<34+0$ weeks $^{\text {b }}$ & $10(31.3)$ & $17(14.3)$ & $3(2.7)$ & $<0.001$ \\
\hline Placental abruption ${ }^{\mathrm{b}}$ & $1(3.4)$ & $3(2.6)$ & $1(0.9)$ & 0.522 \\
\hline Birth weight $(\mathrm{g})^{a}$ & $2520(1076 ; 3215)$ & $3020(2420 ; 3430)$ & $3200(2870 ; 3545)$ & $<0.001$ \\
\hline Gestational age at delivery (completed weeks) & $37(31 ; 39)$ & $39(36 ; 41)$ & $39(39 ; 40)$ & $<0.001$ \\
\hline Delivery by Cesarean section ${ }^{\text {b }}$ & $18(69.2)$ & $56(49.1)$ & $27(23.9)$ & $<0.001$ \\
\hline
\end{tabular}

Data are provided as ${ }^{a}$ median (interquartile range) or ${ }^{b} \mathrm{n}(\%)$ 
Table 3 Multivariate logistic regression models for the prediction of placental insufficiency and intrauterine growth retardation

\begin{tabular}{|c|c|c|c|c|c|c|c|c|c|}
\hline \multicolumn{2}{|c|}{ Parameter } & \multicolumn{2}{|c|}{ Placental insufficiency } & \multirow[t]{2}{*}{ B (se B) } & \multirow[t]{2}{*}{$p$} & \multicolumn{2}{|l|}{ IUGR } & \multirow[t]{2}{*}{$B(S D B)$} & \multirow[t]{2}{*}{$p$} \\
\hline & & Yes $(n=21)$ & No $(n=235)$ & & & Yes $(n=32)$ & No $(n=224)$ & & \\
\hline \multirow[t]{3}{*}{ Group $^{b}$} & Controls & $1(4.8)$ & $112(46.1)$ & reference & 0.001 & $10(31.3)$ & & reference & 0.016 \\
\hline & Intrapl. hematoma & $9(42.9)$ & $20(8.2)$ & $4.20(1.17)$ & & $8(25.0)$ & & $1.81(0.63)$ & \\
\hline & Retropl. hematoma & $11(52.4)$ & $103(42.4)$ & $2.93(1.11)$ & & $14(43.8)$ & & $0,94(0.52)$ & \\
\hline \multicolumn{2}{|c|}{ Parity $(n)^{a}$} & $1(0 ; 2)$ & $2(0 ; 2)$ & $-0.45(0.30)$ & 0.133 & $1(0 ; 2)$ & $2(0 ; 2)$ & $-0.45(0.24)$ & 0.065 \\
\hline \multicolumn{2}{|c|}{ Age (years) ${ }^{a}$} & $32(26 ; 38)$ & $31(26 ; 35)$ & $0.08(0.04)$ & 0.069 & $30(23 ; 34)$ & $31(27 ; 35)$ & $-0.02(0.04)$ & 0.660 \\
\hline \multicolumn{2}{|l|}{$\mathrm{IGDM}^{\mathrm{b}}$} & $3(14.3)$ & $48(20.4)$ & $-0.22(0.72)$ & 0.755 & $6(18.8)$ & $45(20.1)$ & $-0.18(0.58)$ & 0.759 \\
\hline \multicolumn{2}{|l|}{$\mathrm{PIH}$} & $4(19.0)$ & $20(8.2)$ & $-0.41(0.98)$ & 0.676 & $4(12.5)$ & $20(8.9)$ & $-0.25(0.85)$ & 0.772 \\
\hline \multicolumn{2}{|c|}{ Preeclampsia ${ }^{b}$} & $3(14.3)$ & $11(4.5)$ & $2.21(1.17)$ & 0.059 & $3(9.4)$ & $11(4.9)$ & $0.55(0.99)$ & 0.582 \\
\hline \multicolumn{2}{|c|}{ Previous IUGR } & 0 & $8(3.4)$ & $-17.90(12,437.91)$ & 0.999 & $6(18.8)$ & $2(0.9)$ & $4.02(0.99)$ & $<0.001$ \\
\hline \multicolumn{2}{|c|}{ Previous IUFD ${ }^{b}$} & $2(9.5)$ & $5(2.1)$ & $0.76(1.00)$ & 0.448 & $1(3.1)$ & $6(2.7)$ & $-0.02(1.16)$ & 0.986 \\
\hline \multicolumn{2}{|c|}{ Constant } & - & - & $-7.14(1.85)$ & 0.000 & - & - & $-1.93(1.11)$ & 0.082 \\
\hline
\end{tabular}

Patients with second-trimester miscarriage were excluded from these analyses

Data are provided as ${ }^{a}$ median (interquartile range) or ${ }^{b} \mathrm{n}(\%)$

Abbreviations: Intrapl. intraplacental, Retropl. retroplacental, IUGR intrauterine growth retardation, IGDM insulin-dependent gestational diabetes mellitus,

$P I H$ pregnancy-induced hypertension, IUFD intrauterine fetal death

(Table 4), both intra- and retroplacental hematomas, lower parity and presence of IUGR increased the risk significantly (women with IUFD excluded). When taking intra- and retroplacental locations together, the presence of hematoma was also associated with a significantly increased risk for early preterm delivery $(\beta=2.00 \pm 0.63$, $p=0.001)$.

Then, the impact of gestational age at hematoma diagnosis on pregnancy outcome was assessed. Women with placental insufficiency revealed a higher median gestational age at diagnosis (23 completed weeks, IQR 15-27) than those without (15 weeks, IQR 12-24; $p=0.034$ ), whereas there was no difference between women with and without IUGR (median 21 weeks, IQR 12-24, versus median 15, IQR 12-25, respectively; $p=0.851$ ). Median gestational age at diagnosis was higher in women with early preterm delivery (23 weeks, IQR 14-27) than in patients who delivered after $34+0$ weeks $(14$, IQR $12-$ $24 ; p=0.038)$. When dividing women into those with a first-trimester diagnosis of hematoma $(n=70)$ versus those with a second-trimester diagnosis $(n=73)$, placental insufficiency was more frequent after a second- $(15 / 73$, $20.5 \%)$ than after a first-trimester diagnosis $(5 / 70,7.1 \%$; $p=0.029)$. The same was found for early preterm delivery

Table 4 Multivariate logistic regression models for the prediction of early preterm delivery $<34+0$ weeks

\begin{tabular}{|c|c|c|c|c|c|}
\hline \multicolumn{2}{|c|}{ Parameter } & \multicolumn{2}{|c|}{ Early preterm delivery } & \multirow[t]{2}{*}{$\beta($ se $\beta)$} & \multirow[t]{2}{*}{$p$} \\
\hline & & Yes $(n=26)$ & No $(n=224)$ & & \\
\hline \multirow[t]{3}{*}{ Group $^{b}$} & Controls & $3(11.5)$ & $110(49.1)$ & reference & 0.001 \\
\hline & Intrapl. hematoma & $10(38.5)$ & $19(8.5)$ & $2.92(0.78)$ & \\
\hline & Retropl. hematoma & $13(50.0)$ & $95(42.4)$ & $1.81(0.70)$ & \\
\hline \multicolumn{2}{|c|}{ Parity $(n)^{a}$} & $1(0 ; 2)$ & $2(0 ; 2)$ & $-0.63(0.27)$ & 0.021 \\
\hline \multicolumn{2}{|c|}{ Age (years) ${ }^{a}$} & $31(26 ; 35)$ & $31(27 ; 35)$ & $0.04(0.03)$ & 0.283 \\
\hline \multicolumn{2}{|l|}{$\mathrm{IGDM}^{\mathrm{b}}$} & $2(7.7)$ & $47(21.0)$ & $-0.98(0.82)$ & 0.377 \\
\hline \multicolumn{2}{|l|}{$\mathrm{PIH}^{\mathrm{b}}$} & $3(11.5)$ & $21(9.4)$ & $-1.45(1.07)$ & 0.235 \\
\hline \multicolumn{2}{|c|}{ Preeclampsiab } & $3(11.5)$ & $11(4.9)$ & $1.81(1.12)$ & 0.105 \\
\hline \multicolumn{2}{|l|}{$I U G R^{b}$} & $8(30.8)$ & $23(10.3)$ & $1.27(0.56)$ & 0.023 \\
\hline \multicolumn{2}{|c|}{ Previous IUGR ${ }^{\mathrm{b}}$} & 0 & $8(3.6)$ & $-19.06(12,628.30)$ & 0.999 \\
\hline \multicolumn{2}{|c|}{ Previous IUFD ${ }^{\mathrm{b}}$} & $2(7.7)$ & $5(2.2)$ & $0.71(1.01)$ & 0.486 \\
\hline \multicolumn{2}{|c|}{ Constant } & - & - & $-4.40(1.39)$ & 0.001 \\
\hline
\end{tabular}

Patients with second-trimester miscarriage and IUFD were excluded from this analysis

Data are provided as ${ }^{a}$ median (interquartile range) or ${ }^{b} \mathrm{n}(\%)$

Abbreviations: Intrapl. intraplacental, Retropl. retroplacental, IUGR intrauterine growth retardation, IGDM insulin-dependent gestational diabetes mellitus,

$P I H$ pregnancy-induced hypertension, IUFD intrauterine fetal death 
(patients with IUFD excluded: $18 / 71,25.4 \%$ versus $5 / 66$, $7.6 \%$, respectively; $p=0.006)$ but not for IUGR (14/73, $19.2 \%$ versus $8 / 70,11.4 \%$, respectively; $p=0.249$ ).

\section{Discussion}

This retrospective study provided the following key findings: (i) both intra- and retroplacental hematomas were associated with increased incidences of pregnancyrelated complications, including placental insufficiency, intrauterine growth retardation, and preterm labor and (early) preterm delivery. These findings are underlined by the differences in birth weight and gestational age at delivery between the groups; (ii) women with an intraplacental hematoma were at an even higher risk for these complications than those with a retroplacental hematoma. In contrast, only the latter type of hematoma was associated with IUFD in our study population; (iii) retroplacental hematomas revealed a significantly earlier onset than intraplacental hematomas; and (iv) intraplacental hematomas were an independent risk factor for the development of placental insufficiency and IUGR.

Undoubtedly, intrauterine hematomas put the affected women at an increased risk for pregnancy-related complications. The question is whether differentiating hematomas according to their exact position would be justified in order to provide adequate information about associated risks. One might argue that the accurate position of a hematoma has only descriptive value, because there were no relevant differences reported in outcome according to a previous publication [1]. However, we are the first to describe intraplacental hematomas as a separate entity in a clinical setting. According to the results of our study, pregnancies complicated by intraplacental hematomas would carry a different risk profile than pregnancies with a retroplacental hematoma. In particular, the risks for placental insufficiency, growth retardation, preterm labor, and (early) preterm delivery were significantly higher for women with intraplacental hematomas. Notably, in multivariate analyses, intraplacental hematomas were an important risk factor for the development of placental insufficiency and growth restriction (Table 3) as well as for early preterm delivery (Table 4). In intraplacental hematomas, most of the blood is reportedly maternal [7]. However, these hematomas likely form a leak in the fetal circulation as well. Thus, fetal anemia, and, consequently, underperfusion might result. Particularly in the case of profound placental bleeding, a massive reduction in placental function can occur $[7,15]$. This also fits the observation that intraplacental hematomas were significantly larger than those in a retroplacental location. This should also be considered in the context of the size of retroplacental hematomas. Those retroplacental hematomas large enough to cause adverse prenatal effects on the fetus may also produce secondary abnormalities, including intraplacental bleeding [7]. In this case, patients would have been assigned to the intraplacental hematoma group in our study.

Notably, whether the size of an intrauterine hemorrhage would correlate with a worse pregnancy outcome, and therefore, could be used as a predictive marker, has been previously evaluated. However, the results did not reach significance $[3,16]$. This might have been due to the fact that the exact location of the hematoma exact location was not considered.

Preterm labor was quite common in women with intraplacental hematomas (Table 2). It has already been suggested that these bleeding events are accompanied by the release of cytokines [7]. This could trigger preterm labor. However, retro- but not intraplacental hematomas were associated with a high rate of premature preterm rupture of membranes. We consider this to be due to either cytokine release or the fact that retroplacental bleeding, when it extends along the decidua basalis and reaches the placental margin, might lead to local irritation of the amnion.

Notably, cases of IUFD occurred only in the retroplacental hematoma group. We believe that this was due to an "all-or-none" phenomena. Retroplacental hematomas seemed to have developed significantly earlier, which is suggested by the lower gestational age at initial diagnosis (median 13 vs. 24 weeks). In these earlier weeks of gestation, significant impairment of fetal perfusion more likely leads to IUFD. One might also argue that smaller, and, thus, more irrelevant retroplacental hematomas that developed early did not cause symptoms, such as vaginal bleeding, and, consequently, remained undiagnosed. It has already been suggested that many retroplacental hematomas are easily overlooked [7]. This possible bias could also explain the high IUFD rate in the retroplacental hematoma group.

We would like to provide the following hypotheses to explain the fact that intraplacental hematomas obviously developed later in pregnancy than retroplacental hematomas. (i) As suggested previously, subchorionic/retroplacental hemorrhage might be the result of abnormal development of the placental membranes in the first trimester [17]. In contrast, the blood of intraplacental hematomas is mostly maternal and intervillous circulation is not fully established at the end of the first trimester, suggesting that this hematoma entity must develop later in pregnancy $[7,18]$. (ii) The source of bleeding could also be linked to trophoblastic activity that leads to extensive expansion of the spiral ateries' lumina, beginning with the second trimester. Accordingly, the massive intraplacental hematomas could develop only from this gestational age on. Moreover, it has already been mentioned that rounded intraplacental haematomas form as a result of disruption of vasculopathic 
decidual arterioles in a setting of maternal vascular underperfusion and are thus etiologically distinct from retroplacental hematomas [6].

Thus, if a hematoma is found early in pregnancy, i.e. within the first 21 weeks of gestation, its location will likely be retroplacental, whereas intrauterine hematomas found at a higher gestational age will be intraplacental in the majority of cases. However, gestational age at diagnosis will not predict hematoma location with apodictic certainty, since the presented median gestational ages at diagnosis were associated with quite a big interquartile range. Moreover, it has been mentioned that gestational age at diagnosis might predict pregnancy risks $[19,20]$. However, we consider hematoma type of higher predictive value for pregnancy complications than age at diagnosis; women with a second-trimester diagnosis which is associated with the finding of an intraplacental hematoma in the majority of cases carried the highest risks.

The retrospective nature of our study may have introduced some kind of selection bias, as discussed above, and thus, must be considered a study limitation. We cannot provide exact data on changes in sonographic presentation of the hematomas which has to be considered a study limitation. From our clinical experience, hematomas did not get absorbed during follow-up, but their sonographic appearence changed to a more inhomogeneous structure with varying degrees of echogenicity. Neither we are able to provide data about histo -/pathological examinations of the affected placentae. Only a minority of the placentae have been sent for pathological examination, a limitation that is associated with the retrospective study design. Moreover, in case of retroplacental hematomas such an examination seems of only minor impact, since these hematomas are striped off easily. However, we present the first clinical outcome data that could potentially differentiate intra- and retroplacental hematomas.

\section{Conclusions}

Intra- and retroplacental hematomas have different risk profiles for the affected pregnancy. While the rate of IUFD was highest in women with retroplacental hematomas, intraplacental hematomas put the fetus at a significant risk for growth retardation. Clinicians should be aware of these different risk profiles. Prospective studies are warranted to confirm our observations and evaluate adapted treatment strategies.

\section{Abbreviations}

IUFD: Intrauterine fetal death; IUGR: Intrauterine growth restriction
Availability of data and materials

The authors do not wish to share the data due to the missing patients permission to publish (which is not necessary and unusual for retrospective studies at the Medical University of Vienna).

\section{Authors' contributions}

All authors substantially contributed to the manuscript: JO: the project's and the manuscript's conception and design, acquisition of data, statistical analyses, drafting the article and revising it for intellectual content, final approval of the version to be published. PP: the project's and the manuscript's conception and design, drafting the article and revising it for intellectual content, final approval of the version to be published. RP: the project's and the manuscript's conception and design, statistical analyses, drafting the article and revising it for intellectual content, final approval of the version to be published. SP: acquisition of data, drafting the article and revising it for intellectual content, final approval of the version to be published. JW: drafting the article and revising it for intellectual content, final approval of the version to be published. KCM: the project's and the manuscript's conception and design, acquisition of data, drafting the article and revising it for intellectual content, final approval of the version to be published.

\section{Ethics approval and consent to participate}

The study was approved by the Institutional Review Board of the Medical University of Vienna (IRB number: 1681/2014). Neither written nor verbal informed consent is unnecessary in retrospective studies according to the Ethics Committee of the Medical University of Vienna and was, thus, not obtained.

\section{Competing interests}

The authors declare that they have no competing interests.

\section{Publisher's Note}

Springer Nature remains neutral with regard to jurisdictional claims in published maps and institutional affiliations.

\section{Author details}

${ }^{1}$ Clinical Division of Gynecological Endocrinology and Reproductive Medicine, Medical University of Vienna, Waehringer Guertel 18-20, 1090 Vienna, Vienna, Austria. ${ }^{2}$ Department of Obstetrics and Fetomaternal Medicine, Medical University of Vienna, Waehringer Guertel 18-20, 1090 Vienna, Vienna, Austria. ${ }^{3}$ Second Department of Internal Medicine, Klinikum Wels-Grieskirchen, Wagnleithnerstraße 27, 4710 Grieskirchen, Upper Austria, Austria. ${ }^{4}$ Department of Obstetrics and Gynecology, Saint John of God Hospital Eisenstadt, Johannes-von-Gott Platz 1, 7000 Eisenstadt, Burgenland, Austria.

Received: 18 March 2016 Accepted: 6 October 2017

Published online: 26 October 2017

\section{References}

1. Leite J, Ross P, Rossi AC, Jeanty P. Prognosis of very large first-trimester hematomas. J Ultrasound Med. 2006;25:1441-5.

2. Pearlstone M, Baxi L. Subchorionic hematoma: a review. Obstet Gynecol Surv. 1993;48:65-8.

3. Børlum KG, Thomsen A, Clausen I, Eriksen G. Long-term prognosis of pregnancies in women with intrauterine hematomas. Obstet Gynecol. 1989; 74:231-3.

4. Seki H, Kuromaki K, Takeda S, Kinoshita KN. Persistent subchorionic hematoma with clinical symptoms until delivery. Int J Gynaecol Obstet. 1989;63:123-8.

5. Nagy S. Clinical significance of subchorionic and retroplacental hematomas detected in the first trimester of pregnancy. Obstet Gynecol. 2003;102:94-100.

6. Fitzgerald B, Shannon P, Kingdom J, Keating S. Rounded intraplacental haematomas due to decidual vasculopathy have a distinctive morphology. J Clin Pathol. 2011;64:729-32.

7. Kraus FT, Redline RW, Gersell DJ, Nelson DM, Dicke JM. Placental pathology. Atlas of non-tumor pathology; 2004. p. 117-57.

8. American College of Obstetricians and Gynecologists. Task force on hypertension in pregnancy. Report of the American College of Obstetricians and Gynecologists' task force on hypertension in pregnancy. Obstet Gynecol. 2013;122:1122. 
9. ACOG practice bulletin. Diagnosis and management of preeclampsia and eclampsia. Obstet Gynecol. 2002;99:159-67.

10. Neerhof MG, Thaete LG. The fetal response to chronic placental insufficiency. Semin Perinatol. 2008;32:201-5.

11. Schaffer $H$, Steiner $H$, Staudach A. Reference values for qualitative and quantitative analysis of uterine, fetoplacental and fetal flow velocity waveforms. J Fertil Reprod. 1998;2:12-3.

12. Chalubinski KM, Repa A, Stammler-Safar M, Ott J. Impact of Doppler sonography on intrauterine management and neonatal outcome in preterm fetuses with intrauterine growth restriction. Ultrasound Obstet Gynecol. 2012;39:293-8.

13. Mercer BM. Preterm premature rupture of the membranes. Obstet Gynecol. 2003;101:178-93.

14. ACOG practice bulletin no. 127. Management of preterm labor. Obstet Gynecol. 2012;119:1308-17.

15. Kaplan C, Blanc WA, Elias J. Identification of erythrocytes in intervillous thrombi: a study using immunoperoxidase identification of hemoglobins. Hum Pathol. 1982;13:554-7.

16. Ball RH, Ade CM, Schoenborn JA, Crane JP. The clinical significance of ultransonographically detected subchorionic hemorrhages. AJOG. 1996;174: 996-1002.

17. Jauniaux E, Johns J, Burton $\mathrm{G}$. The role of ultrasound imaging in diagnosing and investigating early pregnancy failure. Ultrasound Obstet Gynecol. 2005; 25:613-24.

18. Jaffe $R$, Jauniaux E, Hustin J. Maternal circulation in the first-trimester human placenta-myth or reality? AJOG. 1997;176:695-705.

19. Xiang L, Wei Z, Cao Y. Symptoms of an intrauterine hematoma associated with pregnancy complications: a systematic review. PLoS One. 2014;9(11):e111676.

20. Maso G, D'Ottavio G, De Seta F, Sartore A, Piccoli M, Mandruzzato G. Firsttrimester intrauterine hematoma and outcome of pregnancy. Obstet Gynecol. 2005;105:339-44.

\section{Submit your next manuscript to BioMed Central} and we will help you at every step:

- We accept pre-submission inquiries

- Our selector tool helps you to find the most relevant journal

- We provide round the clock customer support

- Convenient online submission

- Thorough peer review

- Inclusion in PubMed and all major indexing services

- Maximum visibility for your research

Submit your manuscript at www.biomedcentral.com/submit 